
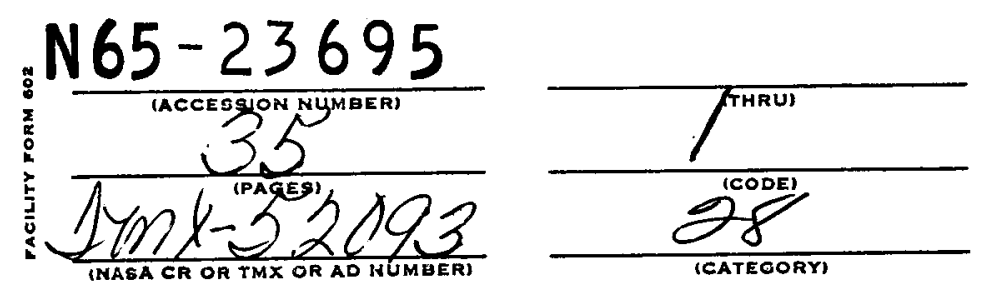

\title{
ENVIRONMENTAL EFFECTS ON LABORATORY AND IN-FLIGHT PERFORMANCE OF NEUTRALIZATION SYSTEMS
}

by J. M. Sellen, Jr. and Ronald J. Cybulski Lewis Research Center Cleveland, Ohio

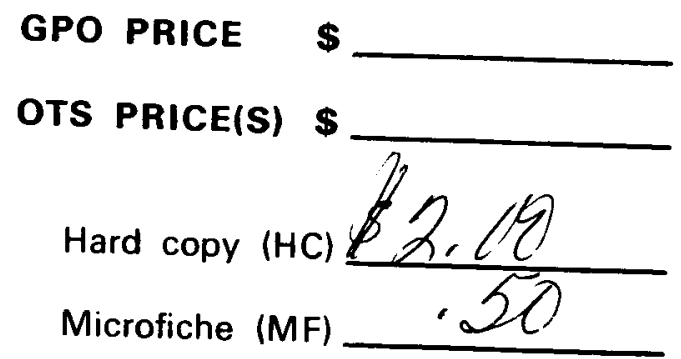

TECHNICAL PREPRINT prepared for Second Aerospace Sciences Meeting of the American Institute of Aeronautics and Astronautics New York, New York, January 25-27, 1965 AIAA Paper No. 65-70 


\title{
ENVIRONMENTAL EFFECTS ON LABORATORY AND IN-FLIGHT PERFORMANCE OF NEUTRALIZATION SYSTEMS
}

\author{
by J. M. Sellen, Jr. and Ronald J. Cybulski \\ Lewis Research Center \\ Cleveland, Ohio
}

TEC HINICAL PREPRINT prepared for

Second Aerospace Sciences Meeting sponsored by the American Institute of Aeronautics and Astronautics

New York, New York, January 25-27, 1965 


\section{ENVIRONMENTAL EFFECTS OF LABORATORY AND IN-FLIGHT \\ PERFORMANCE OF NEUTRALIZATION SYSTEMS* \\ by J. M. Sellen, Jr. \\ Thompson Ramo Wooldridge \\ Space Technology Laboratories \\ Redondo Beach, California \\ and}

Ronald J. Cybulski

Lewis Research Center

National Aeronautics and Space Administration

Cleveland, Ohio

\section{INTRODUCTION}

The neutralization of the high perveance ion beams required for electrically propelled spacecraft has been a subject of continuing interest in the development of these ion thrustors. This neutralization has been examined in laboratory environments in the earlier experiments and, more recentiy, in the first space flight test of an ion thrustor. Effective neutralization of the ion stream has been demonstrated in this latter testing condition as well as in the laboratory experiments. This paper will review environmental effects as they have influenced experiments on ion thrust beams in three particular environmental configurations. These configurations are the small chamber (4 m) neutralization experiments with the electron-bombardment mercury ion thrustor, the large chamber $(25 \mathrm{~m})$ experiments with the contact ionization cesium ion thrustor, and the flight test of the electronbombardment engine on the NASA SERT I spacecraft. The discussion will also review some of the diagnostic techniques that have been employed and will consider further diagnostic techniques, particularly those applicable for space flight tests of ion thrustors.

\section{SMALL CHAMBER NEUTRALIZATION EXPERIMENTS: ELECTRON-}

\section{BOMBARDMENT ION THRUSTOR}

The results of the neutralization tests of this electron-bombardment thrustor have been described in detail in Refs. 1 and 2 , and the discus-

*This work supported by NASA Lewis Research Center, Cleveland, Ohio under Contract NAS3-4114. 
sion here will be primarily concerned with environmental differences between this laboratory testing array and the true space condition. Two of the more obvious features present in the laboratory, and absent in the vehicular space environment, are the conducting boundaries to the vacuum testing chamber and the residual gas within. The conducting boundaries support electric fields through induced surface charges, absorb particles from the plasma stream, and, to some measure, emit secondary particles. The residual gas may interact with the ions and electrons in the thrust beam, with ion-atom charge exchange and electronatom elastic and inelastic scattering being the principal interaction me hanisms.

The neutralization experiments with the mercury electron-bombardment ion thrustor (Kaufman engine) were carried out in a vacuum chamber approximately 4 meters in length and 2 meters in diameter. The test configuration is shown in Fig. I. The designation of a "small" chamber is to distinguish this experimental array from the "large" chamber configuration ( $8 \mathrm{~m}$ in diameter, $25 \mathrm{~m}$ in length) to be discussed in Section III. Both the diameter and the length of the vacuum chamber are of interest. The diameter of the chamber provides the radial withdrawal of the chamber boundaries from the plasma beam, with a coupling between the plasma beam and the boundaries which diminishes for an increased chamber diameter. The length of the vacuum chamber, in turn, provides the axial withdrawal of the collecting boundaries from the ion source, and, for increased chamber lengths, increases the time during which the plasma is isolated from the collecting boundaries in the pulsed-beam tests of the neutralization system.

The pulsed-beam technique is a method of isolating the plasma thrust beam from its near environment, thereby minimizing the role of the boundaries to the testing chamber. Its initial use is described in Ref. 3. A further use of this technique is given in Ref. 4. In the pulsed beam studies, the ion acceleration voltage is pulsed on rapidly, and the neutralization of the ion stream is studied during the initial time-of-flight of the ions across the source to collector interspace. During this period there is no direct absorption of particles by the boundaries, so that the role of the surfaces is reduced to, at most, the termination of electrical lines of force from the plasma stream. This "displacement current" contribution is monitored, in turn, by induction grids that line the testing chamber.

The role of the residual gas in the testing chamber may be minimized, or eliminated, through the maintenance of the chamber below the "critical" pressures. The critical pressure in terms of the charge exchange interaction is that at which the mean free path for charge exchange between the ions in the thrust beam and the residual atoms becomes comparable to the source-to-collector spacing. For the charge exchange of mercury ions on mercury atoms, this mean free path is of the order of 4 meters at pressures of $2 \times 10-5$ Torr, and for lower 
chamber pressures than this, the charge exchange interaction is of diminishing importance. The "critical" pressure in terms of electronatom elastic scattering interactions is that at which the mean free path for large angle scatterings becomes of the order of the dimensions of the plasma stream. For scattering cross sections of the order of 10-16 square centimeter, this mean free path is of the order of 1 meter at $3 \times 10^{-3}$ Torr. The critical pressure in terms of electron-atom inelastic collisions is somewhat less well defined but would, presumably, be that pressure at which energy loss rates for electrons in these collisions become comparable with the rates of energy losses (or gains) from ion-electron interactions in the plasma stream. There has not been, however, a thorough experimental examination of the energy interchange mechanisms between ions and electrons in these plasma thrust beams. In the experiments to be discussed in Section III, the electron-ion interaction appears to lead to a rapid cooling of the electrons after their injection into the ion stream. If this condition should hold true generally, then the pressure requirements in terms of these inelastic interactions are less stringent than those of charge exchange and elastic scattering.

In the experiments detailed in Refs. 1 and 2, the chamber pressure was maintained at $1 \times 10^{-5}$ Torr or less. At full beam currents of $\sim 400$ milliamperes, the use of large liquid-nitrogen-cooled cryopumping surfaces was required to hold the chamber pressure in the range below $1 \times 10^{-5}$ Torr. From this, it may be seen that any substantive interaction between the plasma stream and the residual gas has been eliminated, and the reduction of the pressure to the residual backgrounds in space should not produce any significant variations in the observed behavior of the thrust beam.

The electrical interaction between the plasma beam and the chamber boundaries was similarly small and may be neglected. For those sections of the plasma column near the ion source, the capacitance per unit length between the plasma beam and the chamber walls is 20 micromicrofarad per meter, leading to an induced charge of 20 micromicrocoulombs per meter per volt of potential difference between the plasma column and the boundaries. For a full beam current of 400 milliamperes at 2500-electron-volt ion energy, the positive charge per unit length in the plasma beam is $8 \times 10^{6}$ micromicrocoulombes per meter. By proper adjustment of the plasma potential it is possible for the induced charge along the boundaries to become $\sim 10^{-5}$ of the magnitude of the ion and electron charge colonies in the plasma stream. In the regions downstream from the ion source, this isolation is less complete because of the outward divergence of the plasma column (from electrostatic lens effects in the ion acceleration region). This radial spreading of the ion beam diminishes the separation from the plasma to the boundaries with a corresponding increase in the capacitance between the thrust beam and the vacuum chamber 
walls. However, by using a chamber of moderate diameter, the induced charge per unit length remains less than $10^{-3}$ of the ions and electrons per unit length in the plasma column, even in these downstream, diverged beam regions.

The results of the neutralization tests with the mercury electronbombardment thrustor, taken from Ref. 2, are shown again in Fig. 2. For the neutralizer configuration that was later used in the space flight test, the plasma potential, measured at a downstream point, was $\sim 9$ volts positive with respect to the neutralizer. These potential differences are taken from emissive probe data, with an accuracy of measurement of $\sim 0.5$ volt in that experimental condition. Significantly, there were no observable differences between the pulsed beam operation of the neutralizer and the steady-state condition that one obtained when the plasma beam was directed against a floating collector. For neutralizers that are withdrawn from the plasma column, observable differences do occur between these two periods, but for totally immersed (and thus, tightly coupled) neutralizers, the behavior is identical in the two testing conditions. This is further evidence, then, of a neutralization system which is well-coupled to the plasma stream.

In view of the intimate coupling between the neutralizer and the plasma beam, and in view of the very minor involvement of the chamber boundaries and the residual gas in the behavior of the plasma beam, there is no a priori reason to consider that the neutralization of the thrust beam would be adversely affected if the chamber walls were removed to infinity and the chamber residual gases reduced to the almost total vacua of space. There are, however, limitations in this small chamber experiment relative to a true space test. The most notable is that the plasma column is isolated from its laboratory environment for periods of the order of 200 microsecond or less, and, if some particular relaxation processes do occur over longer periods they would not have been observable in this 4-meter geometry. Beyond this, there are other factors such as the absence in the testing chamber of the dilute residual plasmas that exist in the near regions of the Earth, and the more complicated general interaction between the plasma thrust beam and the magnetic field of the Earth, when the thrust beam is exhausted from a moving vehicle. While these latter factors are not easily amenable to laboratory simulation, the extension of the isolation time of the plasma column from its vacuum testing chamber may be carried out through the use of larger testing facilities. These experiments were performed in the large testing chamber at Lewis Research Center, and their results are now discussed. 


\section{LARGE CHAMBER NEUTRALIZATION EXPERIMEITTS: CONTACT}

\section{IONIZATION CESIUM ION THRUSTOR}

The large testing chamber at NASA Lewis Research Center, Cleveland, Ohio has a diameter of $\sim 8$ meters and a length of $\sim 25$ meters. For a pulsed beam experiment, this chamber length would provide, for nominal ion accelerating voltages, an isolation time almost an order of magnitude larger than that attained in the smaller chamber experiments discussed in Section II. Relaxation processes in the millisecond range would be observable, if, indeed, processes with these long-time constants should exist in these plasma columns.

Because the diagnosis would be directed at the possible existence of second-order variations in the plasma column behavior as functions of the plasma column length, a contact ionization source was selected for the ion beam generation. The experiments are an extension of the earlier tests described in Ref. 3. The plasma produced by the contact ionization source is very quiescent, and measurements of plasma potential and electron temperature may be performed with an accuracy that is subject only to the inherent limitations of the diagnostic instruments. For potential measurements with emissive probes, this accuracy is of the order of 0.01 to 0.03 volt in the plasma density region above $10^{7}$ ions per cubic centimeter, ${ }^{5}$ while the Langmuir probe accuracy in the measurement of electron temperatures is estimated at $\sim 200^{\circ} \mathrm{K}$. The ion source perveance was 2000 nanopervs, so that the neutralization experiments were conducted with a "broad" beam, high perveance thrustor. Neutralization was by an immersed hot wire.

The experimental array used in these large chamber experiments is shown in Figs. 3 and 4. These figures are drawn from Ref. 6, which presents in detail the results of the experiments. The important aspect of these tests, applied to the present discussion, was the invariance in the plasma behavior through these longer isolation periods. The potential, the density, and the electron temperature at a fixed probe location in the plasma column exhibited no observable timewise variations as the plasma front moved further downstream from the probe. For those probe locations near the ion source, the time interval from the arrival of the plasma front at the probe until the plasma arrived at the collector at the far end of the chamber is greater than 1 millisecond. This result then confirms that there are no significant relaxation processes with characteristic times of the order of a millisecond.

The plasma columns obtainable even in this testing configuration are not, of course, those infinitely long columns that will be obtained in the vehicular space environment. They are of great enough length, however, for the ion density within the plasma column to have diminished to values comparable to the ambient plasma densities in the ionosphere. 
For the average ion beam divergence angle of $\sim 3^{\circ}$ in these cesium ion beams, the plasma density diminishes from lO10 ions per cubic centimeter in the source region to $\sim 10^{6}$ ions per cubic centimeter at points of the order of 20 to 25 meters downstream. Such a beam, then, would have almost merged into the ambient plasma of the F2 layer. 3 For regions of greater altitude, this ambient plasma density diminishes, which requires a longer column length for the thrust beam plasma to merge with the space plasma. These merging points, however, would differ by only an order of magnitude for a wide range of vehicle altitudes, and the existence of very effectively neutralized plasma columns in this present testing geometry is strong evidence that the effectiveness of the neutralization would not be diminished in any way in the infinite geometries of space. The conclusive evidence, of course, would be supplied through a vehicular space test of an ion engine. This space test was performed and is described in the following section.

IV. NEUTRALIZATION EXPERIMENTS IN SPACE:

SERT I ION ENGINE TEST

The SERT I spacecraft was launched on July 20, 1964, from Wallops Island, Virginia. This flight test was under the direction of the INASA Lewis Research Center. The four-stage Scout launch vehicle boosted the payload into a ballistic trajectory with a peak altitude of 2100 nautical miles and with a total flight time of $\sim 40$ minutes above 250 nautical miles. The spacecraft possessed both a cesium contact ionization thrustor and an electron-bombardment mercury ion thrustor. The electron-bombardment thrustor was identical to that thrustor which was tested in the experiments described in Section II, and it is the neutralization behavior of this thrustor that will be discussed in this section.

A detailed treatment of the flight package and of the space flight results is given in Refs. 8 and 9. The discussion here will review briefly the methods by which the thrust and the beam power were determined, and will then consider, in more detail, the information furnished by the rotating-vane electric field strength meter.

\section{A. Thrust and Beam Power Measurements}

The SERT I spacecraft is shown in Fig. 5. The payload was spin stabilized with the spin induced by the fourth stage rocket. After the deployment of the ion thrustors, the spin rate of the vehicle was -85 revolutions per minute about the spin axis. This spin rate was measured by two separate solar detectors. The initial precession angle was small, and further damping occurred during the flight period. 
The thrust of the electron-bombardment engine acted to increase the spin rate of the vehicle. The measured change in the spin period of the vehicle from the solar detectors, together with the known moment of inertia of the vehicle about the spin axis and the known moment arm of the thrust beam about the spin axis were used in the calculation of the engine thrust. A further measure of the angular acceleration of the vehicle from a radial accelerometer allows an additional determination of the engine thrust. The peak value of this delivered thrust was 6 millipounds, and the total increment increase in the vehicle spin was $10 \mathrm{rpm}$.

The measured parameters of the ion thrustor include discharge current and discharge potential in the electron-bombardment chamber, arc chamber magnetic field current, ion acceleration voltage, total ion source current, accelerator drain current, and neutralizer current. From these measured currents and voltages, the deliverable thrust from the ion engine, assuming perfect neutralization, may be calculated. With suitable corrections for the fraction of doubly charged ions, the average divergence angle of the accelerated ions, and the spurious drain currents from the neutralizer to the ion source region, 9 this calculated thrust, when perfect neutralization is assumed, is in agreement with the measured values of the thrust within 5 percent.

This close agreement between the calculated and measured values of thrust is conclusive evidence that ion beams can be effectively neutralized in the infinite geometries of space. The quantity which is specifically determined by this experiment is illustrated in Fig. 6, and is the net ion acceleration velocity $\left[2 e\left(v_{0}-V_{p}\right) / M_{4}\right]^{1 / 2}$, where $V_{O}$ is the ion source voltage and $V_{p}$ is the potential in the plasma thrust beam. The possible 5-percent difference in measured and calculated thrust leads to a possible range of 10 percent in the net ion acceleration energy $e\left(V_{0}-V_{p}\right)$. For the employed ion source voltage of $2.5 \mathrm{kilovolts,} \mathrm{this} \mathrm{10-percent} \mathrm{range} \mathrm{corresponds} \mathrm{to} \mathrm{a} \mathrm{possible} \mathrm{range}$ of 250 volts in the potential of the plasma relative to the neutralizer. In the experiments described in Section II, this plasma potential has been determined by emissive probe measurement to $\sim 0.5$ volt, so that the space flight data, though it provides an assurance that the neutralization is perfect within, at most, effects of second order, does not act as a direct check against the laboratory measurements of plasma potential. Instruments that will provide such a direct check for in-flight performance are discussed in Section $V$.

A further check on the effectiveness of the ion beam neutralization is provided by the hot-wire power density probe measurements. This probe, described in Ref. 10, provides a two-dimensional map of the ion beam power density in the ion thrust beam. Te total beam power measurements from this probe agree with the calculated total beam 


\section{B. Rotating-Vane Electric-Field Strength Meter}

\section{General considerations}

The rotating-vane E-field meter consisted of 3 stationary vanes which were alternately shielded and exposed by a set of 3 similar vanes that rotated at $8000 \mathrm{rpm}$. The instrument is similar to that described in Ref. 11. The induced current that flows to the surface of the stator vanes if an electric field is present on the surface of the vehil provides a 400 cps signal which is amplified by a solid-state alternatingcurrent amplifier and then commutated into the telemetry unit. For this vane rotation speed, the induced current density is $3.5 \times 10^{-11}$ amperes per square centimeter for an imposed electric field strength of 1 volt per centimeter. For the amplification provided on the most sensitive scale the minimum field strength that may be detected is $\sim 2$ volts per centimeter.

The rotating-vane E-meter is sensitive to some degrading effects that may occur even in the laboratory environment. One such effect arises if a contact potential difference exists between the forward surface of the stator vanes and the back surface of the rotor vanes. For a vane spacing of 3 millimeters, a contact potential of 1 volt between these two surfaces would produce an effective field strength of $\sim 3$ volts per centimeter when the stator vanes are shielded by the rotor. For the SERT I E-field meter, these vanes are gold plated to minimize such contact potential fields. However, it is possible for these fields to exist, and conditions under which they have been generated are described in Section IV.B.4 (p. 12).

Of more general concern is the difficulty in relating the potential of an isolated vehicle to the surface field strength indicated by the meter, if the vehicle is within the dilute plasma near the Earth. For a vehicle within a perfect vacuum, the vehicle potential is

$$
V_{s}=E_{s} r_{S}
$$

where $E_{S}$ is the surface field strength and $r_{S}$ is the radius of the (spherical) vehicle. For a vehicle in a dilute plasma, however, the near termination of lines of electric force upon plasma particles, rather than at infinity as in the perfect vacuum case, causes a higher electric field strength to exist for a given vehicle potential. The correction factor for this effect may be almost an order of magnitude for small vehicle potentials and relatively dense ambient plasmas, such as those encountered in the F2 layer. Values of this correction are given in Ref. 12 for a series of densities and kinetic temperatures in the ambient plasma. For large vehicle potentials, the sheath region surrounding the vehicle becomes very large, and the correction terms diminish. 
A further difficulty created by the presence of the ambient plasma occurs when the velocity of the vehicle begins to exceed the thermal velocities of the ions in the space plasma. For vehicles in the lower portions of the ionosphere, the condition $\left.v_{S} \gg v_{+}\right|_{\text {thermal }}$ generally

exists. For such a circumstance, a wake structure exists about the vehicle, and the sheath dimensions and electric fields may vary significantly from one portion of the vehicle to another. This condition is illustrated in Fig. 7. Under such a condition, an E-meter on a vehicle that possesses both a spin and a motion through the plasma would sense a time-varying electric field, even for a fixed vehicle potential. Examples of these sheaths and electric fields are also given in Ref. 12 .

A final effect from the presence of a dilute space plasma is the current of charged particles that may flow from the plasma to the vehicle skin. These currents are complicated functions of the vehicle potential, plasma density, plasma thermal energies, and vehicle motions relative to the plasma. For small negative vehicle potentials, the current density of ions from the plasma to the forward portions of the vehicle is $p_{+} v_{S}$, where $\rho_{+}$is the space plasma density and $v_{s}$ is vehicle velocity. This would lead to currents of $10^{-7}$ ampere per square centimeter for the denser regions of the Fo layer ( $10^{6}$ ions $\left(\mathrm{cm}^{3}\right)$. In this same Fe region and for small positive vehicle potentials, the current of electrons may be $\sim 10^{-6}$ ampere per square centimeter because of the greater electron mobility. Large vehicle potentials relative to the space plasma tend to increase the magnitudes of these currents still further. It is important to note that, under particular conditions, these particle currents from the ambient plasma may exceed by many orders of magnitude the induced current density due to electric fields of the order of a few volts per centimeter. For this reason, a signal from the E-meter may be predominantly the result of real particle currents and not the result of the displacement currents associated with the surface electric field. For the SERT I E-field meter, the sensitivity to these particles was diminished by a factor of $\sim 10$ through the use of gridded stator vanes. Tests relating to the E-meter behavior when particle currents are being delivered to the stator vanes are reviewed in Section IV.B.4 (p. 12).

One further environmental factor that may affect the E-meter behavior is the solar ultraviolet radiation. Any photoelectrons liberated from the stator vanes would contribute to the overall current signal treated by the amplifiers. For a solid stator and rotor vane construction, this photoelectric signal should be easily describable because of being suppressed for a positive vehicle potential, being present for a negative vehicle potential, and being absent from any areas of the stator vanes in the rotor vane shadow. The circumstances for a gridded stator vanes placed before a solid backing plate and with a gridded rotor vane are much more involved as is illustrated in 
Fig. 8. Because of these complications, the E-meter response to ultraviolet radiation was examined experimentally. These tests are also reviewed in Section IV.B.4.

2. Observed E-meter signals: flight data

There are four particular periods during the SERT I space test in which the E-meter signal will be discussed. These are the following:

(1) Thrust beam off

(2) Thrust beam on, no neutralizer bias, upper part of trajectory

(3) Thrust beam on, neutralizer biased positive with respect to vehicle skin, upper part of trajectory

(4) Thrust beam on, no neutralizer bias, re-entry portion of trajectory

The E-meter signal from these four periods is shown in Fig. 9. Several features are prominent. During the first period there is, essentially, no signal from the E-meter. During the second period the signal is observable and is periodically fluctuating. During the third period the E-meter signal is nonfluctuating at approximately 0.1 of full-scale amplitude on the high sensitivity scale. Finally, in the fourth period, the signal is again fluctuating and is increased in its magnitude from that of the second period.

When the E-meter signal is examined relative to the rotation of the vehicle, it is found that the fluctuating signals in the second and fourth periods have a component of the same period as the vehicle rotation (unpublished data obtained from J.T. Bagwell and W. H. Hawersaat by NASA Lewis Research Center). The dependence of this signal as a function of the orientation of the vehicle is shown in Fig. 10. From these data the E-meter signal may be characterized as "beam dependent," since it is in existence for those periods when a thrust beam is generated by the thrustor and for those periods in which no neutralizer bias exists, having the period of the vehicle rotation.

\section{Environmental effects: flight operation}

The environmental effects discussed in Section IV.B.I (p. 8) may now be examined as they may have contributed to the flight data from the E-meter. In addition, there are other factors, due to the presence of the thrust beam, that should be considered. These several effects are listed in table $I$. The vehicle rotation period is indicated there as $\mathrm{T}_{\mathrm{S}}$. 
TABIE I. - ENVIRONMENTAL EFFECTS

\begin{tabular}{|c|c|c|}
\hline Effect & $\begin{array}{l}\text { Spin- } \\
\text { dependent } \\
\text { signal }\end{array}$ & Period \\
\hline Solar photoelectric & Yes & $\mathrm{T}_{\mathrm{S}}$ \\
\hline Variations in sheath thickness & Yes & $\mathrm{T}_{\mathrm{S}}$ \\
\hline Plasma collection currents & Yes & $\mathrm{T}_{\mathrm{S}}$ \\
\hline $\overrightarrow{\mathrm{V}} \times \overrightarrow{\mathrm{B}}$ forces & Yes & $0.5 \mathrm{~T}_{\mathrm{s}}$ \\
\hline $\begin{array}{l}\text { Thrust beam-resident plasma } \\
\text { interaction }\end{array}$ & No & -- \\
\hline
\end{tabular}

The solar photoelectric currents would produce a spin-dependent signal with a definite phase relation to the solar sensor signal from which the vehicle rotation speed is determined. Particular conditions of vehicle potential could suppress these photoelectric currents and thus eliminate the solar ultraviolet as a contributor to the spindependent signals that have been observed. Variations in the sheath thickness would appear for small vehicle potentials and for vehicle speeds in excess of the ion thermal velocities in the ambient plasma. These variations in sheath dimensions would diminish for increased vehicle potentials and for diminished plasma densities. Plasma collection currents would increase for increased vehicle potentials, but the variations in these currents to an E-meter for a vehicle with a large potential would tend to diminish as the sheath dimensions increased and became more symmetric about the vehicle.

The remaining effects have not been previously discussed and are, for the most part, conjectural. One of these possible interactions is denoted as " $\overrightarrow{\mathrm{V}} \times \overrightarrow{\mathrm{B}}$ forces." In the experiments in Ref. I3, potential gradients along the axis of the plasma stream were observed when the beam was directed against a transverse magnetic field. In the vehicular experiment, these gradients could result in a fluctuating potential at the Injection region of the column (assuming that the far downstream portions of the plasma column must match to the potential of the ambient plasma), which, in turn, could cause the vehicle potential to exhibit a spin-dependent fluctuation. The period for the fluctuation in this instance would be $0.5 \mathrm{~T}_{\mathrm{S}}$.

A final possible interaction, between the thrust beam and the resident plasma, should not cause any spin-dependent fluctuations in 
vehicle potential, since the ambient plasma should be isotropic except in the very near neighborhood of the vehicle. A spin-dependent E-meter signal could result from the interaction between the thrust beam and the ambient plasma, however, if that interaction resulted in the collection, at the vehicle surface, of currents from the ambient plasma. Such a condition might arise, for example, if currents of secondary electrons from the ion accelerator plate enter the plasma thrust beam. Because of their large energy (several $\mathrm{keV}$ ), these electrons are not "bound" to the plasma column as the electrons from the neutralizer are, and they very rapidly leave the thrust beam and are lost in the adjoining ambient plasma. Thus, even though the neutralizer might be capable of perfect neutralization of the thrust beam, an imbalance in the overall currents leaving the vehicle could result. The resultant small positive shifts in the vehicle potential would extract a net current of electrons from the space plasma in order to balance this extraneous current of fast secondaries leaving the accelerator plate. Indeed, any condition that results in a vehicle potential other than the floating potential for the passive vehicle could result in a spindependent current collection signal from the E-meter.

Throughout the discussion it has been assumed that there are no direct currents between the ion engine and the E-meter. This has been assumed because the ion engine and the E-meter are well removed from each other on the spacecraft and because the thrust beam is directed at $180^{\circ}$ to the normal to the E-meter.

Of the several possible effects presented in table I, the photoelectric currents and plasma collection currents could be tested in laboratory experiments. These are reviewed in the next section.

\section{E-Field meter tests}

An E-field meter, identical to that instrument flown on the SERT I flight, was subjected to a series of laboratory tests. These tests included the response of the meter to static and time-varying electric fields, to plasma particle currents, and to ultraviolet radiation. The experiments are described in Ref. 14 and will be reviewed, in part, in this section.

The direct-current field response was similar to that which was supplied with the flight instrument; it provided a full-scale output signal for $\sim 30$ and $\sim 90$ volts per centimeter on the high and low sensitivity channels, respectively. Primary attention was directed, however, toward the response to time-varying fields. Small amplitude fields, from 0.05 to 15 cps were imposed on the meter with circuit gain and phase lag determined. Rolloff in gain began at $\sim 0.5$ cps and was down $\sim 3$ decibels at $1.5 \mathrm{cps}$, which is the spin frequency of the 
SERT I vehicle. Above this point the response fell off at 20 decibels per decade. Phase lag at $1.5 \mathrm{cps}$ was $\sim 40^{\circ}$. The E-meter signal from effects that are correlated with vehicle spin would then possess a phase lag of $\sim 40^{\circ}$ and a reduced gain of $\sim 3$ decibels for the first harmonic component of $1.5 \mathrm{cps}$, with further reductions in gain and greater phase lags for the higher harmonics. Other tests with time-varying fields investigated the response to step functions, linearly increasing and decreasing fields, and "rectified" sine waves.

Another series of tests examined the meter sensitivity to particle currents. The instrument was placed in a "plasma wind tunnel" as described in Ref. 14 and as illustrated in Fig. 1l. The argon ion plasma stream in this tunnel simulates the plasma density and relative motion for a vehicle moving in the regions of the ionosphere below the protonosphere. The particle current density was varied and the sensitivity of the meter to currents of both ions and electrons was examined. When the overall meter potential was placed negative with respect to the streaming plasma, only ions could be collected by the vanes. For this condition, ion particle current densities of $\sim 3 \times 10^{-9}$ ampere per square centimeter were required to produce meter outputs of the general magnitude observed during the second period of the flight described in Section IV.B.2 ( $p .10)$. For this same plasma density, placing the overall meter potential positive with respect to the plasma produced even large E-meter signals because of the higher mobility of the plasma electrons. Increasing the ion particle current density to 10-8 ampere per square centimeter was sufficient to drive the E-meter signal into saturation for either positive or negative potentials of the meter relative to the plasma stream.

The final series of tests examined the meter sensitivity to ultraviolet radiation. The source of this ultraviolet light was a Hinteregger-type source operating in the alternating-current mode with hydrogen gas at 10 Torr. The lamp provides a broad molecular hydrogen continuum with its maximum energy in the range from 3000 to 1700 angstroms, with strong illumination in the spectral region between the photoemission threshold for gold and the point of maximum quantum yield. While the lamp does provide a substantial ultraviolet output, it is not identical to the solar illuminance that was present at the vehicle surface during the SERT I flight. This is not of principal concern, however, since the photoemission of surfaces is such an involved phenomenon and is so critically dependent on surface conditions that the response of the present E-meter may very well not be the same as that of the flight $E$-meter, even if the two were exposed to identical ultraviolet spectra. The general behavior of the E-meter under ultraviolet radiation may be tested though, and this was the intent of the present series of experiments.

The E-meter did exhibit sensitivities to the ultraviolet radiation. These effects could be suppressed by placing strong retarding potential fields on the E-meter. The signals also diminished when strong 
accelerating fields were placed on the E-meter. While this is not the behavior to be expected for photoelectrons liberated from a simple planar surface, the photoelectric currents from gridded structures placed before solid backing structures may be, as discussed in Section IV.B.1. (p. 8), complicated functions of the fields imposed on the surfaces.

A second test of the meter under ultraviolet light utilized an Osram HBO 200 mercury source with a spectral cutoff at 2500 angstroms. The quanta here are of insufficient energy to create photoelectrons, but they were capable of inducing short-lived E-meter signals that may be interpreted as contact potential shifts.

\section{E-Meter flight data: discussion}

In the discussion of the E-meter data, the approach will be to consider a definite model of the vehicle in-flight condition and to examine the consistency of this model against the observed E-meter signals. The model $O$ be discussed is based on five specific aspects in the observed behavior of these neutralized ion beams:

(1) The neutralizer is closely coupled to the plasma in the pulsed beam tests in the small chamber experiments, in which $V_{p}-V_{N}$ is only of the order of a few volts.

(2) There is invariance in the coupling and in the general plasma behavior for increasing lengths of the plasma column as demonstrated in the 25-meter chamber tests.

(3) When perfect neutralization is assumed, the in-flight demonstration of measure thrust is within a few percent of the calculated thrust, which provides assurance that unusual behavior, if any, in the vehicular environement produces only small perturbations.

(4) The measured axial potential decrements along the axis of these thrust beams, are small, being of the order of a few volts for well neutralized beams, and the potentials diminish as they move downstream from the electron injection region.

(5) The general behavior is such that in the region in which the thrust beam plasma density merges into the ambient plasma the only potential decrements that may be sustained are of the order of a few $\mathrm{kT}_{\mathrm{e}} / \mathrm{e}$, where $\mathrm{T}_{\mathrm{e}}$ is the electron temperature. 
This model leads to the potentials sketched in Fig. 12. The potential in the space plasma is chosen as the zero of the potential and is assumed to be everywhere constant.

For the period before ion beam turn-on, the vehicle has been placed at a small negative potn tial to reject the bulk of the electrons that diffuse to the sheath region surrounding the vehicle. This corresponds to period 1 in Section IV.B.2. (p. 10). After ion beam turn-on, this model would place the vehicle at a slight positive potential if there is no bias of the neutralizer relative to the vehicle skin. This corresponds to periods 2 and 4 of Section IV.B.2. The remaining period 3 , in which the vehicle skin was biased several hundred volts negative with respect to the neutralizer, is indicated in Fig. 12(c).

The first effect to be interpreted in terms of this model is the surface field strength, which is the quantity nominally measured by an E-field meter. If the E-meter signal were due to a surface electric field, that signal should be virtually absent in period 1 , should be present with a small amplitude and with possible spin-frequency variations in periods 2 and 4 , and should be larger and essentially steady in period 3. The observed data are consistent with these predictions in periods $I$ and 3 , but the large fluctuating signals observed in periods 2 and 4 are not consistent with the model in Fig. 12 if the signals are to be generated by only surface electric fields.

The second effect to be considered in terms of the model is the generation of E-meter signals by particle currents from the ambient plasma. In period 1, these signals should be small, as the "passive" vehicle is receiving equal ion and electron currents at this floating potential. In periods 2 and 4 the collection currents should be (for the most part) electrons, should be spin dependent (in general), and should have a substantial magnitude compared to currents of ions. These electron currents should be larger in period 4 than in period 2 because the vehicle in this later period had returned to denser portions of the ionosphere. In period 3 the only collection currents should be ions, with a magnitude reduced considerably compared to the electron currents, and should be reasonably independent of the vehicle spin. The predictions of the model are consistent with the observed E-meter signals. Further, by a combination of particle collection currents and surface electric field effects, the E-meter behavior predicted by the model is consistent with the observed E-meter signals in all periods.

The final effect to interpret in terms of the model is photoelectric currents due to the solar ultraviolet. If photocurrents were present and were contributors to the E-meter signals, these contributions should be present in periods 1 and 3 as a spin-dependent signal and absent in periods 2 and 4 . The observed signals differ from 
this predicted behavior in several respects. This lack of agreement can be interpreted either as an inconsistency between the specific model and the possible solar photoeffects, or as a portion of a more general body of evidence from the flight data against the influence of photocurrents. The several aspects of this larger body of evidence against photoemission effects are as follows:

(1) An E-meter signal is absent in period 1 .

(2) A spin-dependent signal is absent in period 3 .

(3) The growth of the signal in period 4 is from its level in period 2 .

(4) The phase of the E-meter signal is relative to the solar detector.

Apart from the specific model in Fig. 12, it is difficult to postulate a positive vehicle potential for periods 1 and 3 . This is particularly true of period 3. For negative vehicle potentials photoelectrons are accelerated away from the surface, and a photoelectric effect, if present, should be observable. Further, a photoeffect, if present, should not be of increasing magnitude, so that photosignals in period 4 should not differ from those in period 2. Finally, the phase of the E-meter signal might be expected to have the peak of the signal when the meter is facing the sun. However, the observed phase (Fig. 10) shows that the peak of the signal occurs when the E-meter is facing almost directly away from the sun, and the minimum occurs when the meter is facing the sun (the discussion here takes into consideration the phase lags of $\sim 40^{\circ}$ at the $1.5 \mathrm{cps}$ frequency of the vehicle spin). A photoeffect with these phase relations can only be explained as being in addition to, but opposing, a larger signal.

While the several aspects listed are evidence against photoelectric effects, it should be emphasized that this discussion was based on photoemission from a planar surface. For gridded surfaces in the near neighborhood of solid surfaces, the possible exchange of photocurrents becomes so involved that no firm conclusions may be made regarding these signals. The laboratory tests, for example, displayed photosignals only for relatively weak electric fields upon the E-meter surfaces. It is possible, thus, that photoeffects do contribute to the overall E-meter signal in periods 2 and 4 .

In summary, the model of Fig. 12 is consistent with the observed E-meter signals if it is assumed that these signals result primarily from particle collection currents in periods 2 and 4 (with some possible photoeffects in these same periods) and primarily from surface electric fields in periods 1 and 3 . The response of the meter in the laboratory tests and the magnitude of the particle currents to be expected at the 
altitudes of the flight test are, in turn, consistent with the observed signals.

\section{V : ADDITIONAL DIAGNOSTIC TECHNIQUES}

\section{A. Surface Field Measurements}

In Section IV.B.I. (p. 8), some of the limitations in surface field measurements for ion engine diagnosis have been detailed. The primary limitation is that the vehicle potential and the surface electric field strength are not simply related if the vehicle is within the dilute plasmas in the near neighborhood of the Earth. Additional complications result if the vehicle speed exceeds the thermal velocities of the ions in this ambient plasma. At greater altitudes, the plasma densities diminish, ion thermal velocities increase (because of the lighter ion masses at these altitudes), vehicle potential becomes more nearly equal to $E_{S} r_{S}$ (for a spherical vehicle), and the use of the vehicle surface field becomes, in general, less subject to environmental perturbations. The operation of the rotating-vane E-field meter, as a specific technique for determining the surface field strength, is also less subject in these higher altitude regions to the environmental effects of plasma particle currents. Solar photoeffects and passible contact potential shifts would remain as possible perturbations to the rotating-vane meter.

A second technique for surface field measurements is the "button" type E-meters. 15 These devices function by means of small emitted and collected currents ( $10^{-3}$ amps or less), but the emitting areas are also small ( $0.1 \mathrm{~cm}^{2}$ ) so that the current density contributing to the meter signal is large compared to any background currents due to photoemission or particle currents from the ambient plasma. As such, the button $E$-meters are less subject to environmental perturbations.

The surface field measurement does not, however, provide a direct determination of the potential in the plasma thrust beam. Indeed, though the relation between surface field and vehicle potential is a simple one for the distant regions of space, the further relaxation of the vehicle surface field to the thrust beam potential may be quite difficult to make for a vehicle in the almost perfect isolation of these very dilute plasmas. Even further, the nature of those remaining environmental factors is uncertain, because they are complicated by the behavior of the solar winds and their interactions with the magnetic fields and ambient plasmas in the region in question. For these several reasons, other diagnostic techniques that provide a direct determination of thrust beam potential should be considered. 


\section{B. Emissive Probe Measurements}

The plasma potential data given in Fig. 2 were obtained with a conventional emissive probe technique with an accuracy of $\sim 0.5$ volt in this application. If the probes are operated in the floating condition, the accuracy of measurement is approximately 1 to 2 volts. A probe suitable for vehicular use and utilizing a driven shield cathode follower circuit for greater frequency response is described in Ref. 16. This emissive probe does provide a direct measurement of the potential in the plasma and possesses a bandwidth capable of detecting potential fluctuations, if these are present in the vehicular operation of an ion engine, of up to the hundred kilocycle range. Because the probe requires immersion in the plasma beam for best operation, its operating lifetime is limited unless provisions are made for its withdrawal and protection except during sampling periods.

One further use of emissive probes is the determination of the potential of vehicles that are still within regions near the Earth. In this configuration the probe is immersed in the ambient plasma and measures the potential of the plasma relative to the vehicle. Special considerations that apply to the probe operation in these dilute plasmas are discussed in Ref. 5.

\section{SUMMARY}

(The effective neutralization of ion thrust beams has been demonstrated in both controlled laboratory experiments and in the NASA SERT I space test; of a mercury electron-bombardment ion thrustor. In the laboratory experiments the exhaust beam potential was 5 to 10 volts positive with respect to the neutralizer depending on the specific neutralizer configuration. The accuracy of this emissive probe measurement is 0.5 volt. In the SERT I space flight test the measured thrust agrees with the calculated thrust, assuming perfect neutralization, within 5 percent. This demonstrates that the lons attain the acceleration energy the $\widetilde{y}$ would achieve under perfect neutralization to within, at most, second-order effects. The absence of unusual behavior in the SERT I space flight test as compared to the laboratory test when properly conducted is assurance that controlled laboratory tests of neutralization systems do provide an adequate examination of the neutralization process.

The diagnostic techniques by which the ion beam thrust was determined were not subject to any particular perturbations of the vehicular environment. The evidence from the flight data and from the subsequent laboratory tests of the rotating-vane electric field strength meter, however, has shown that environmental effects were contributing factors to the signals from this instrument. A second diagnostic technique for surface field strength measurements, the E-meter button, should possess a reduced sensitivity to environmental factors because of the higher 
current density condition in the operation of the meter.

The interpretation of vehicle potential from the surface field strength measurement requires significant corrections because of the ambient plasma in the near neighborhood of the Earth. For regions more distant from the Earth these perturbations should diminish. In both regions, however, surface field strength measurements do not provide a direct determination of the potential in the thrust beam plasma and supplementary diagnostic techniques should be considered. Emissive probes possess a capability of measuring this exhaust beam potential within a fraction of a volt and of following any fluctuations in this potential for frequencies of up to 100 kilocycles. The probes would require withdrawal from the plasma column except for the measurement periods.

REFERENCES

1. Kemp, R. F., Sellen, J. M., Jr., and Pawlik, E. V.: "Beam Neutralization Tests of a light lode Electron Bombardment Engine," AIAA Preprint 2663-62 (November 1962).

2. Kemp, R. F., Sellen, J. M., Jr., and Pawlik, E. V.: Neutralizer Tests on a Flight-Model Electron-Bombardment Ion Thrustor," NASA TN D-1733 (July 1963).

3. Sellen, J. M., Jr., and Kemp, R. F.: "Cesium Ion Beam Neutralization in Vehicular Simulation," ARS Preprint 61-84-1778 (June 1961).

4. Ward, J. W., and Hubach, R. A.: "Neutralization of Ion Beams from Engines of Annular Geometry," ARS J 32, 1730 (November 1962).

5. Sellen, J. M., Jr., and Kemp, R. F.: "Emissive Probe Characteristics in Dilute Plasmas," APS 6th Annual Meeting of the Division of Plasma Physics (November 1964).

6. Sellen, J. M., Jr., and Kemp, R. F., and Hieber, R. H.: "Observations of Neutralized Ion Thrust Beams in the 25-Meter NASA Testing Chamber," Section III.G of NASA CR-53634 (1964).

7. Hanson, W. B.: "Structure of the Ionosphere," Satellite Environment Handbook, edited by F. S. Johnson (Stanford University Press, Stanford, California, 1961), Chap. 2.

8. Gold, H., Rulis, R. J., Maruna, F. A., Jr., and Hawersaat, W. H.: "Description and Operation of Spacecraft in SERT I ion thrustor flight test," NASA TM X-1077, 1965. 
9. Cybulski, R. J., Shellhammer, D. M., Lovell, R. R., Domino, E. J., and Kotnik, J. T.: "Results from SERT I Ion Rocket Flight Test," NASA TN D-2718, 1965.

10. Baldwin, I. V., and Sandborn, V. A.: "Theory and Application of Hot-Wire Calorimeter for Measurement of Ion Beam Power," Electrostatic Propulsion, edited by D. B. Langmuir, E. Stuhlinger, and J.M. Sellen, Jr., (Academic Press, New York, N. Y., 1961), pp. $425-446$.

11. Waddel, R. C.: "An Electric Field Meter for Use on Airplanes," Rev. Sci. Instr. 19, 31, (January 1948).

12. Hall, D. F., Kemp, R. F., and Sellen, J. M., Jr.: "Plasma-Vehicle Interaction in a Plasma Stream," AIAA J. 2, 1032 (June 1964).

13. Sellen, J. M., Jr., and Bernstein, W.: "Interactions of Collisionless Plasma Streams With Transverse Man etic Fields," Phys. Fluids I, 977 (1964).

14. Kemp, R. F., Cole, R. K., Hall, D. F., ane Sellen, J. M., Jr.: "Sensitivity Tests on a Flight-Model Rotating-Vane E-Meter," NASA CR 54147, 1965.

15. Forbes, S. G., Kemp, R. F., Sellen, J. M., Jr., Shelton, H., and Slattery, J. C.: "Ion Engine Testing Techniques," ARS Preprint 2183-61 (October 1961).

16. Fulton, A. S., and Muchmore, R. B.: "Design, Development, and Fabrication of a Floating Emissive Probe for Ion Beam Diagnostics," TRW Space Technology Laboratories, final report Contract NAS3-2517 (June 1963). 


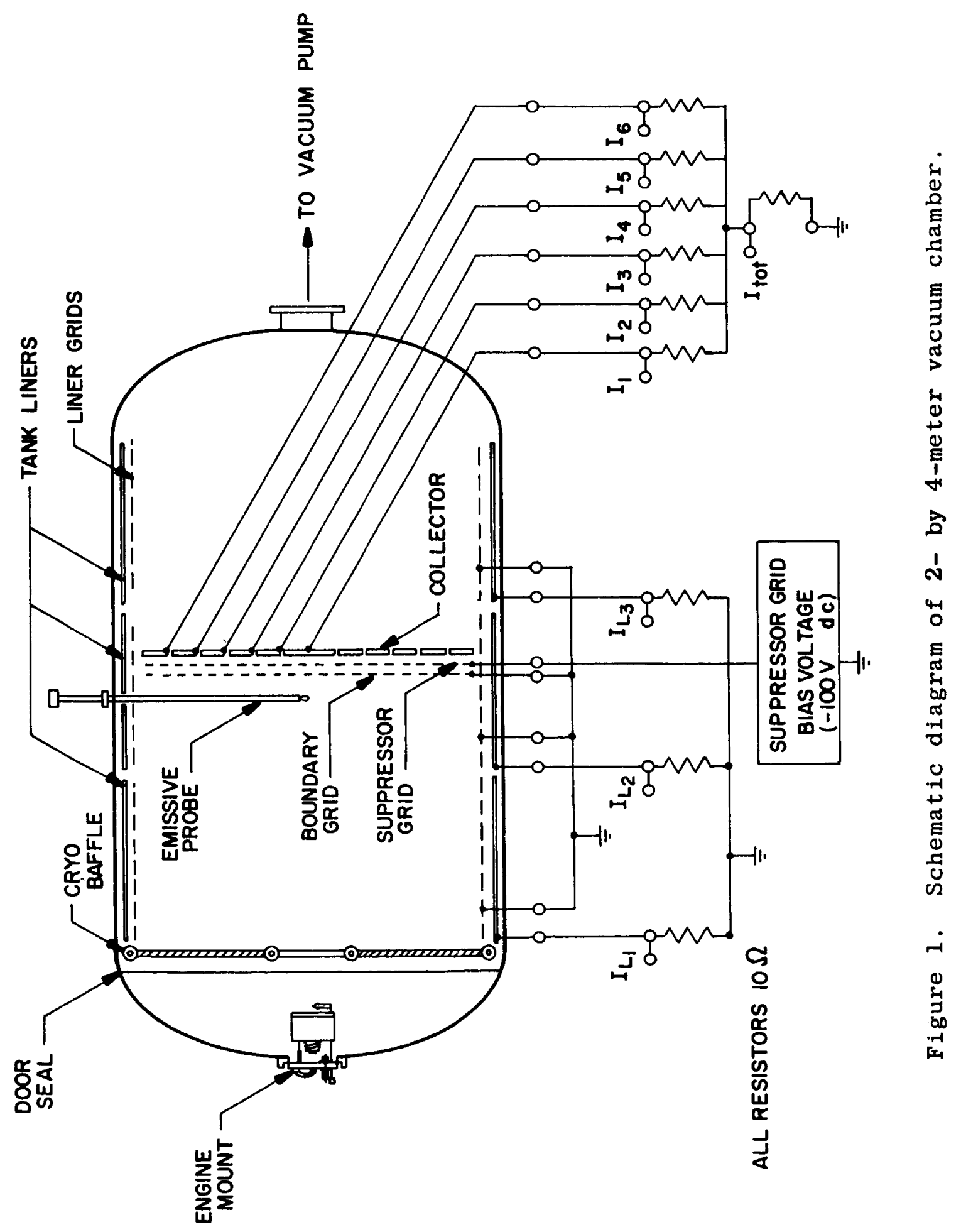




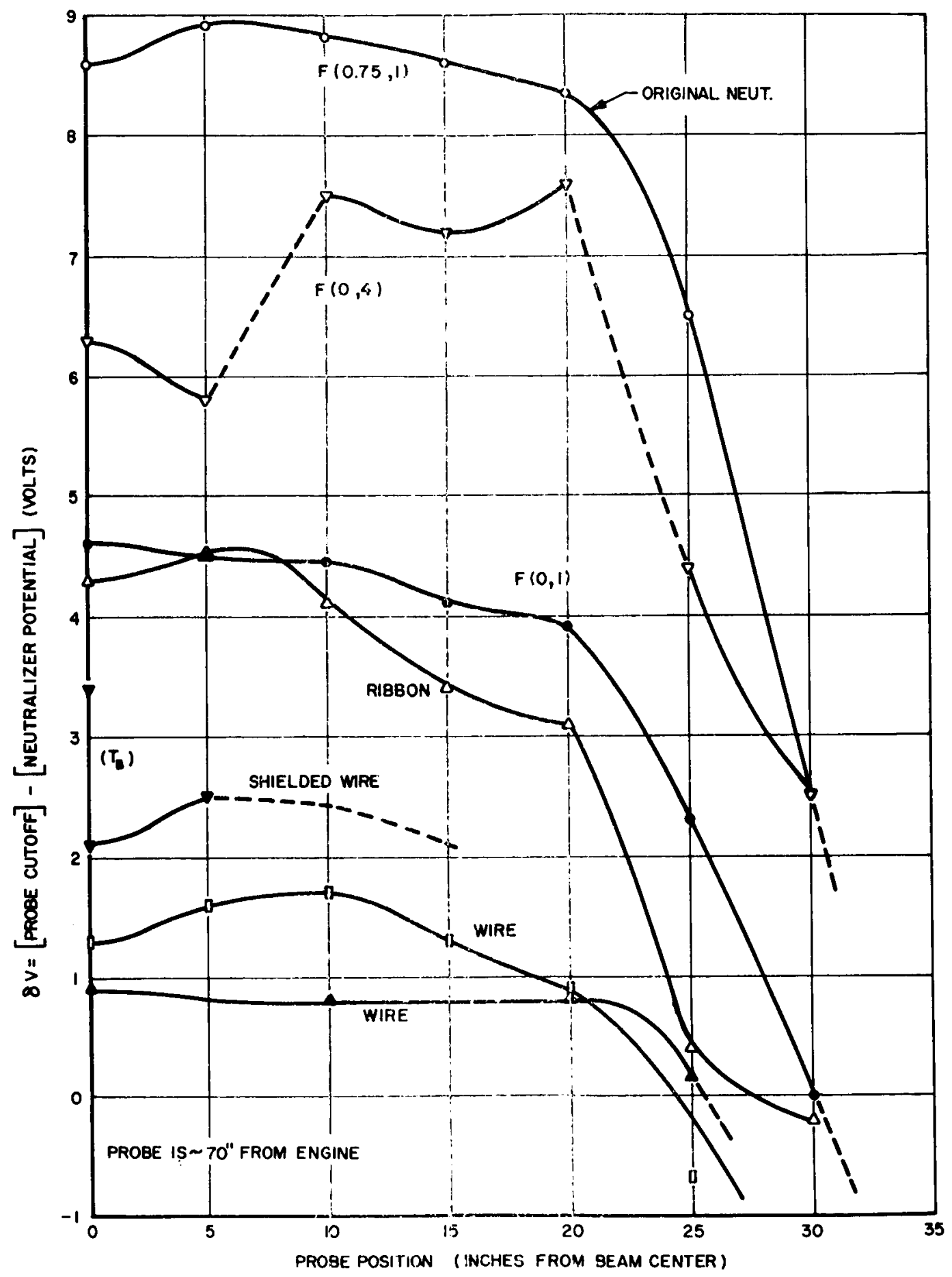

Figure 2(a). - Emissive probe measurement of potential difference between plasma and neutralizer as functions of radial probe position. Axial probe position is 70 inches downstream. F-type neutralizers are of folded-ribbon design used in SERT I thrustor. Numbers in parentheses are radial and axial coordinates of position of neutralizer tip. 


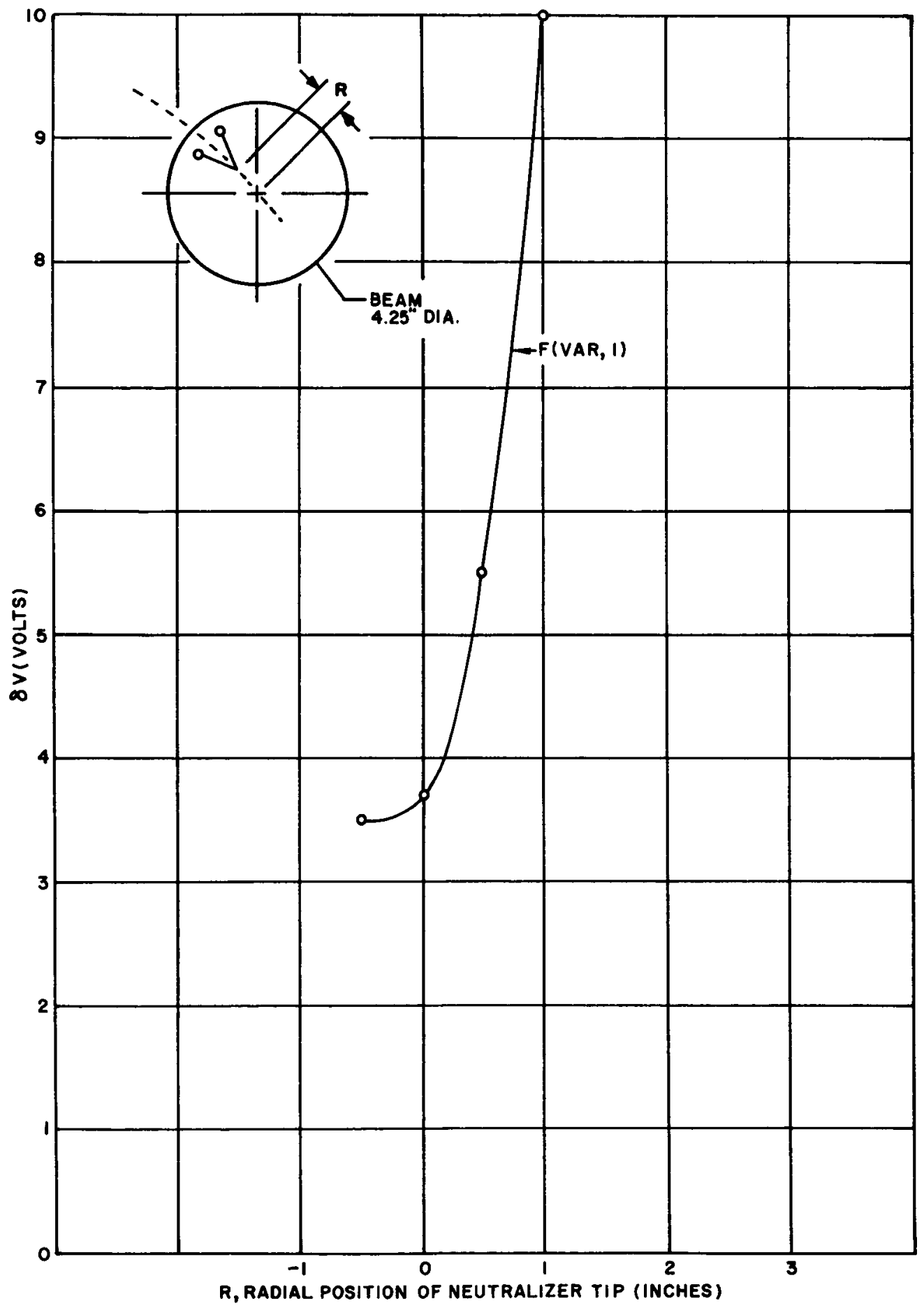

Figure 2(b). - Emissive probe measurements of potential difference between plasma and neutralizer as function of position of the variable-position neutralizer. Probe position is on beam axis 70 inches downstream. F (var, 1) designates flight-type neutralizer of variable radial position; it is located 1 inch downstream from accelerator grid. 


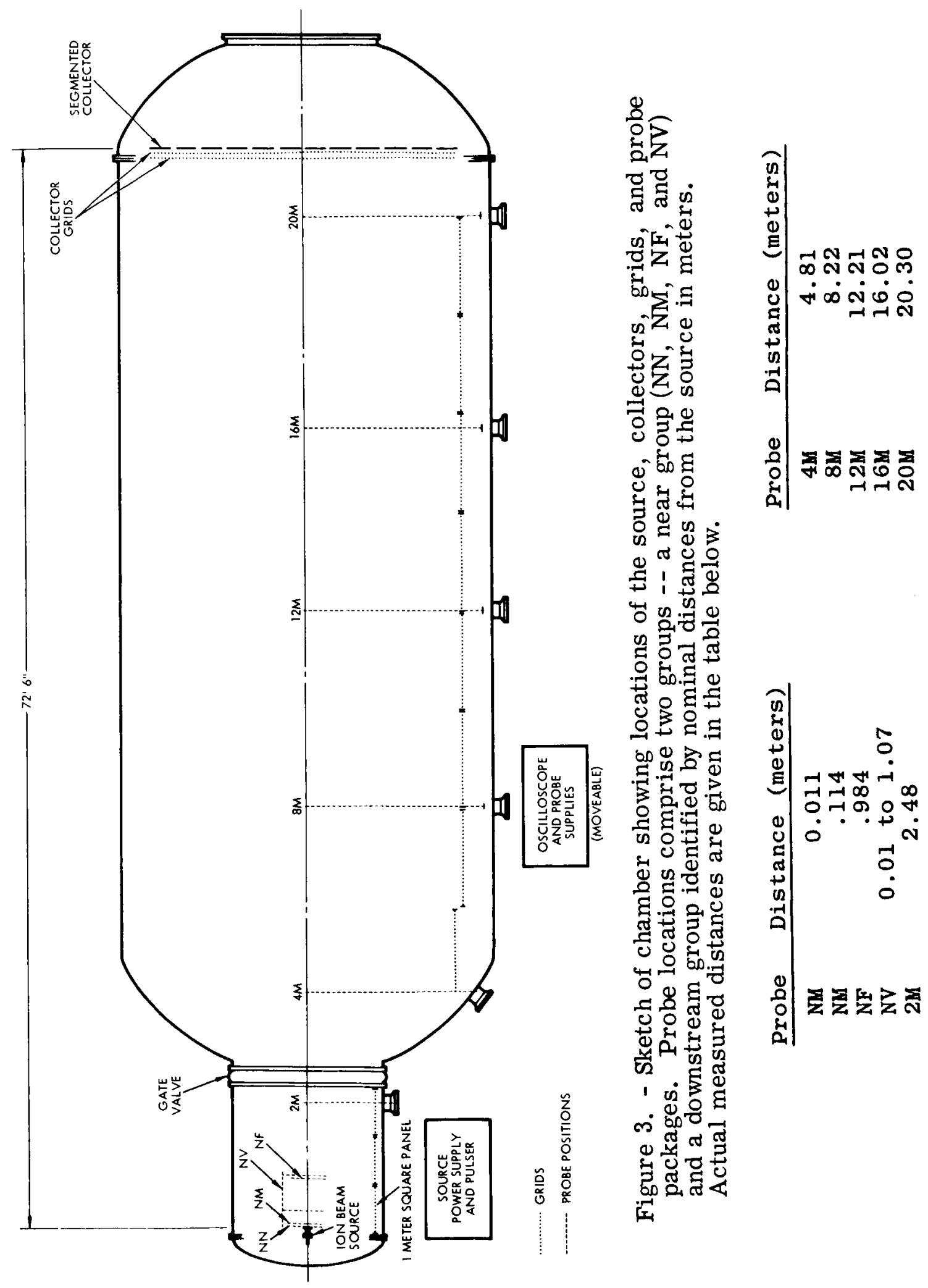




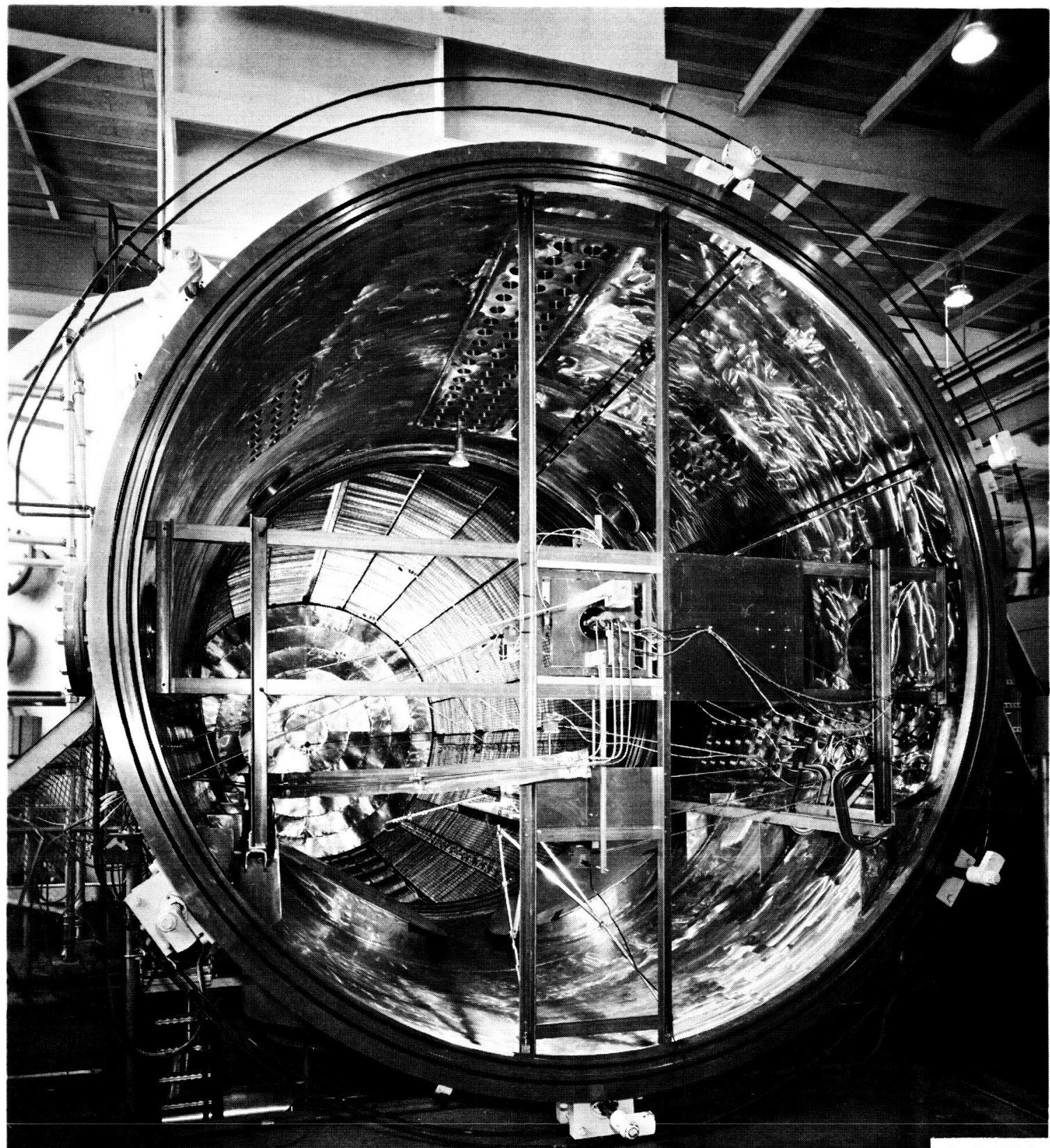

C- 64213

Figure 4. - View through "bell-jar" section into interior of testing chamber. Downstream probe packages are mounted at the end of booms, which are swung from side ports. In the foreground center is the source mounting plate. 


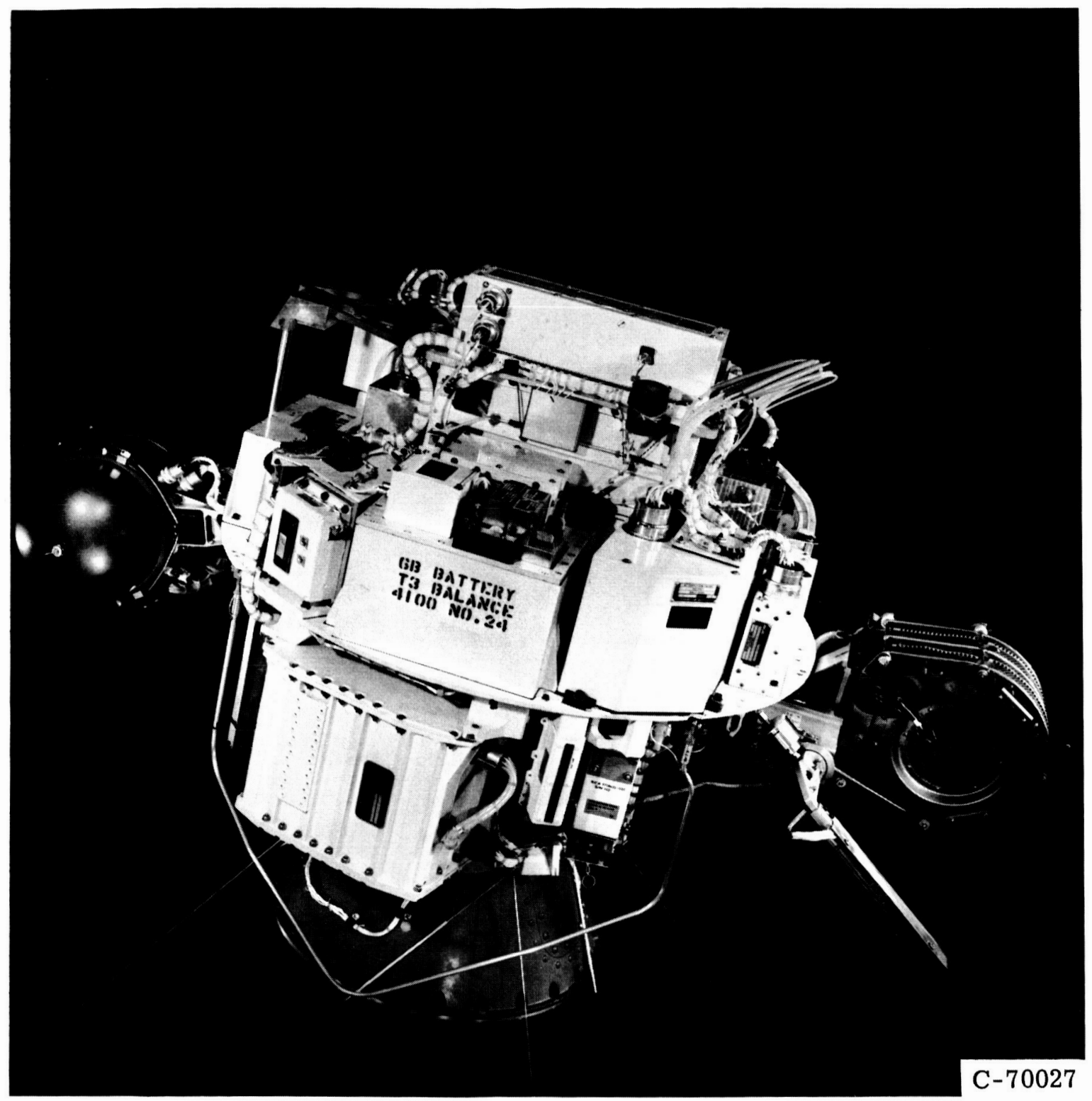

Figure 5. - Free flight configuration of the SERT I spacecraft. 


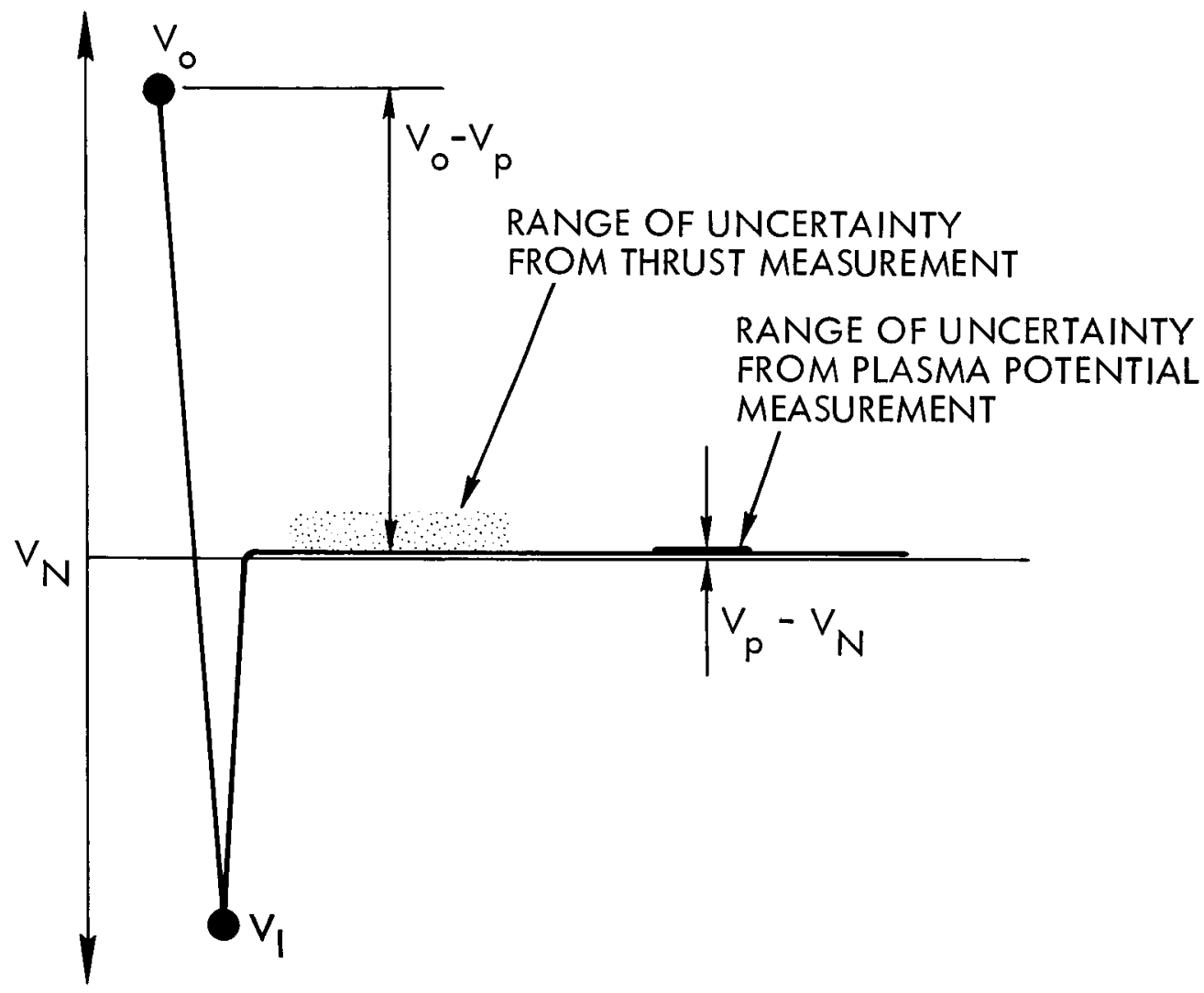

Figure 6. - Flight test measurements of thrust determine ion acceleration velocity $\left[2 e\left(V_{o}-V_{p}\right) / M_{+}\right]^{1 / 2}$ from which $\mathrm{V}_{\mathrm{o}}-\mathrm{V}_{\mathrm{p}}$ is derived. Laboratory plasma potential measurements determine $\mathrm{V}_{\mathrm{p}}-\mathrm{V}_{\mathrm{N}}$. 


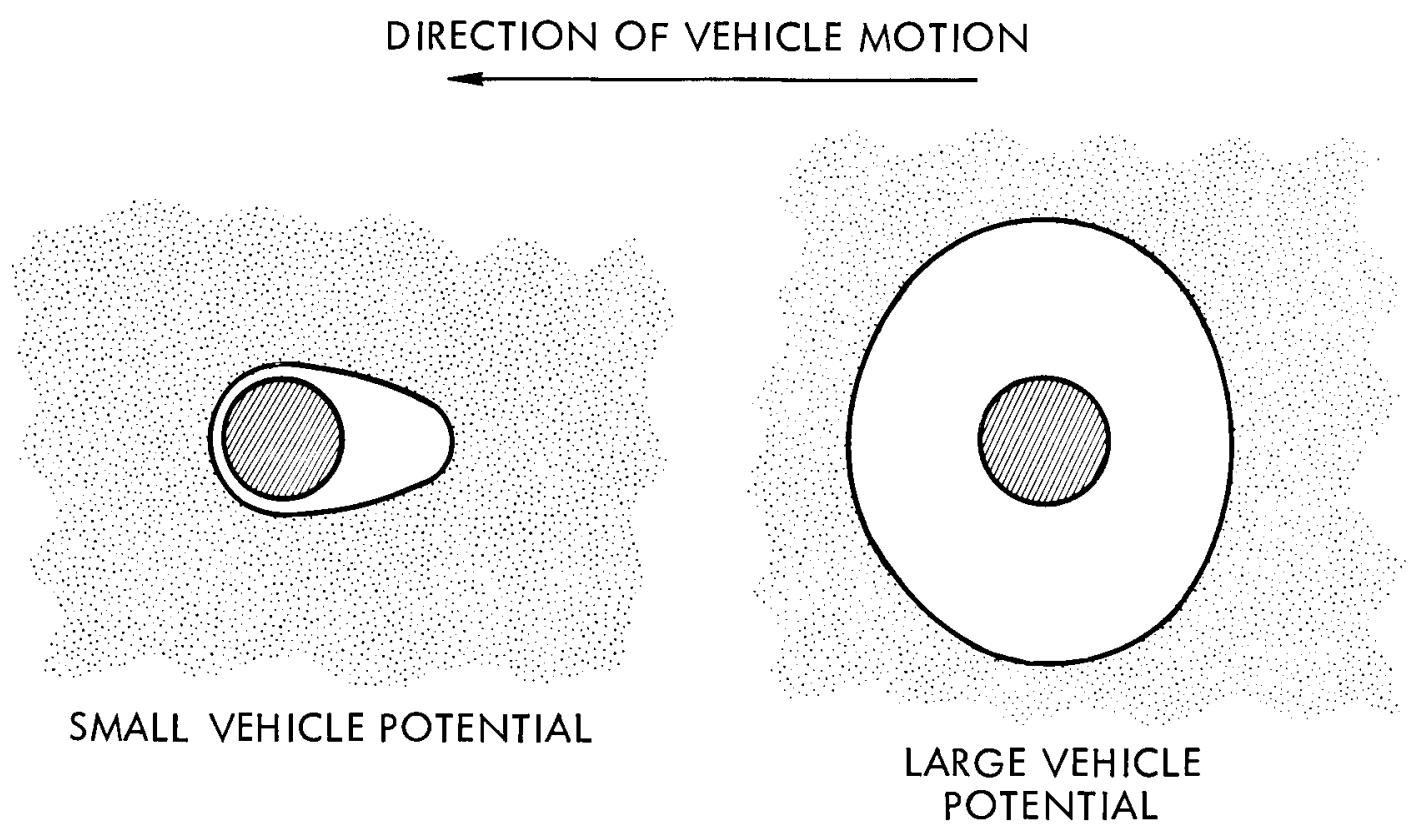

Figure 7. - Sketch of vehicle sheath configuration for "small" and "large" vehicle potentials. 

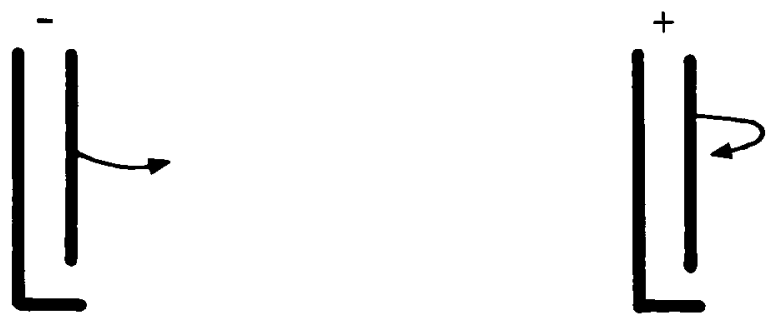

SOLID VANE
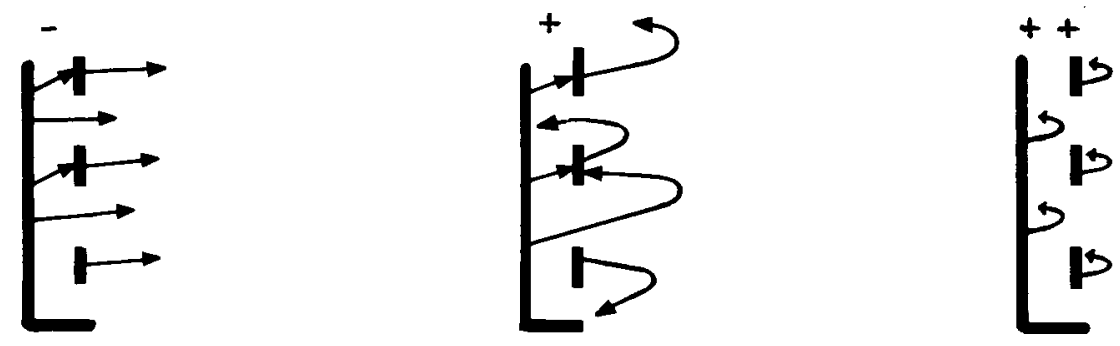

GRIDDED VANE

Figure 8. - Possible photoelectric currents from solid and gridded stator vanes for positive and negative vehicle potentials. Strongly positive potentials are indicated ++ . 
HIGH-SENSITIVITY CHANNEL

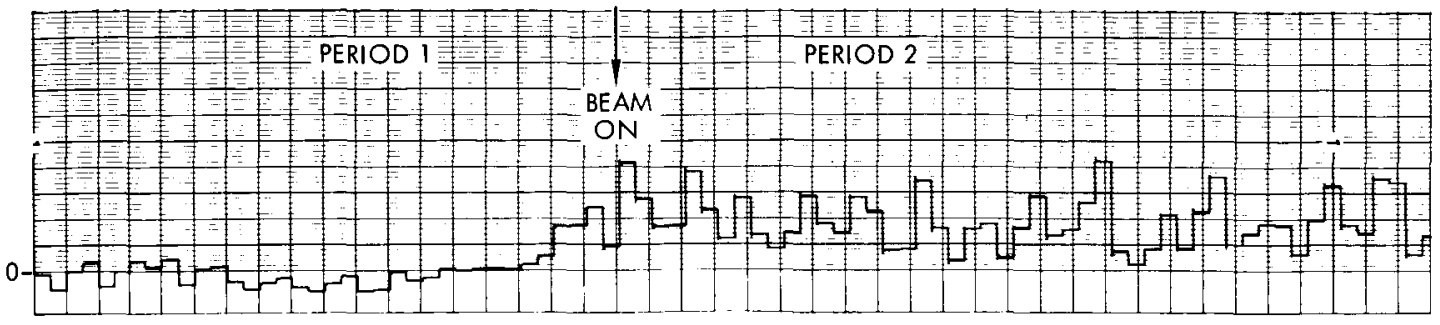

HIGH-SENSITIVITY CHANNEL
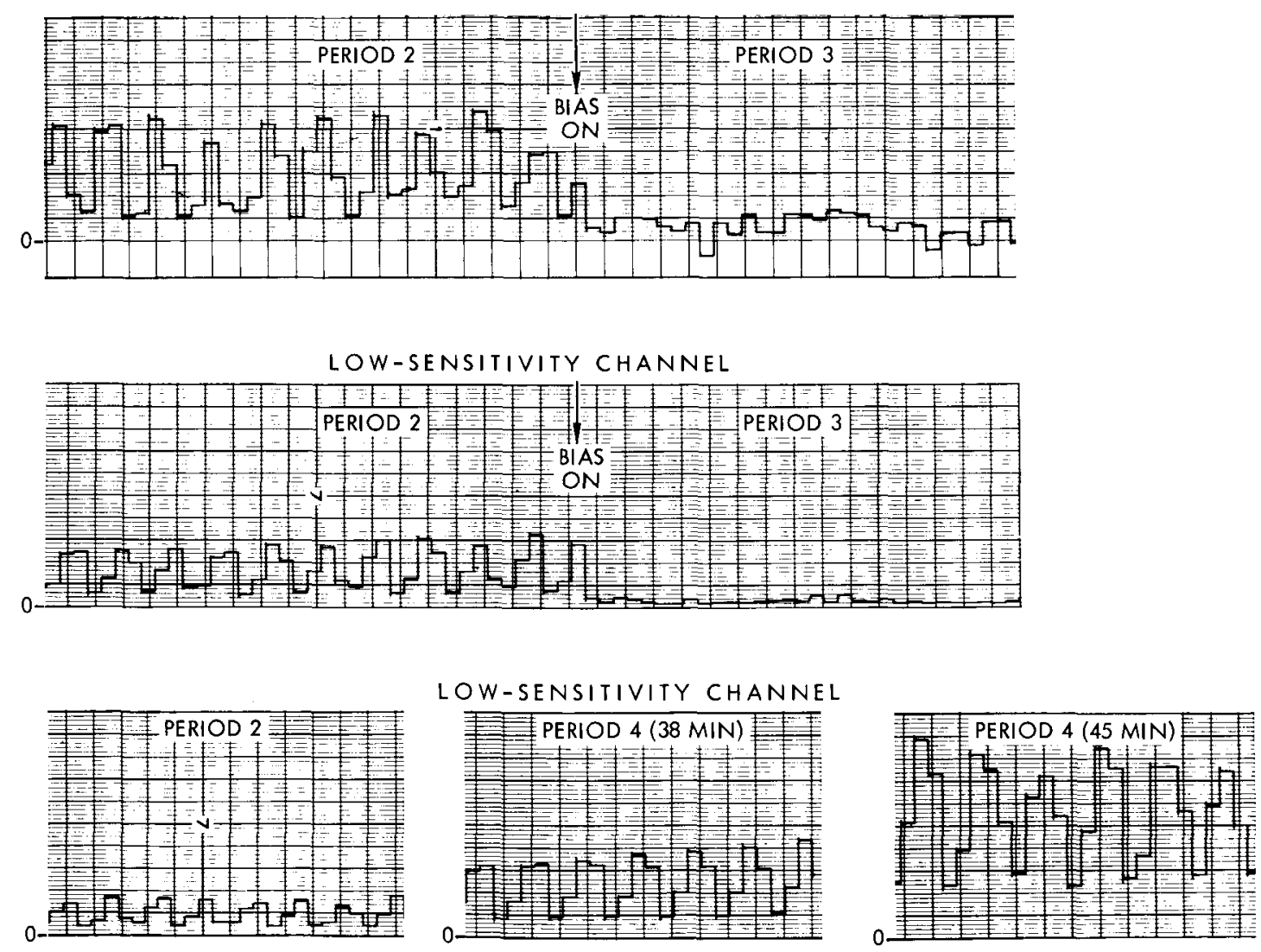

Figure 9. - E-field meter signals during periods 1 to 4 of SERT I space flight test. One horizontal division equals 1 second. Full scale is 10 vertical divisions. Indicated times in period 4 are measured from launch. Data in period 2 are at approximately 20 minutes after launch. 


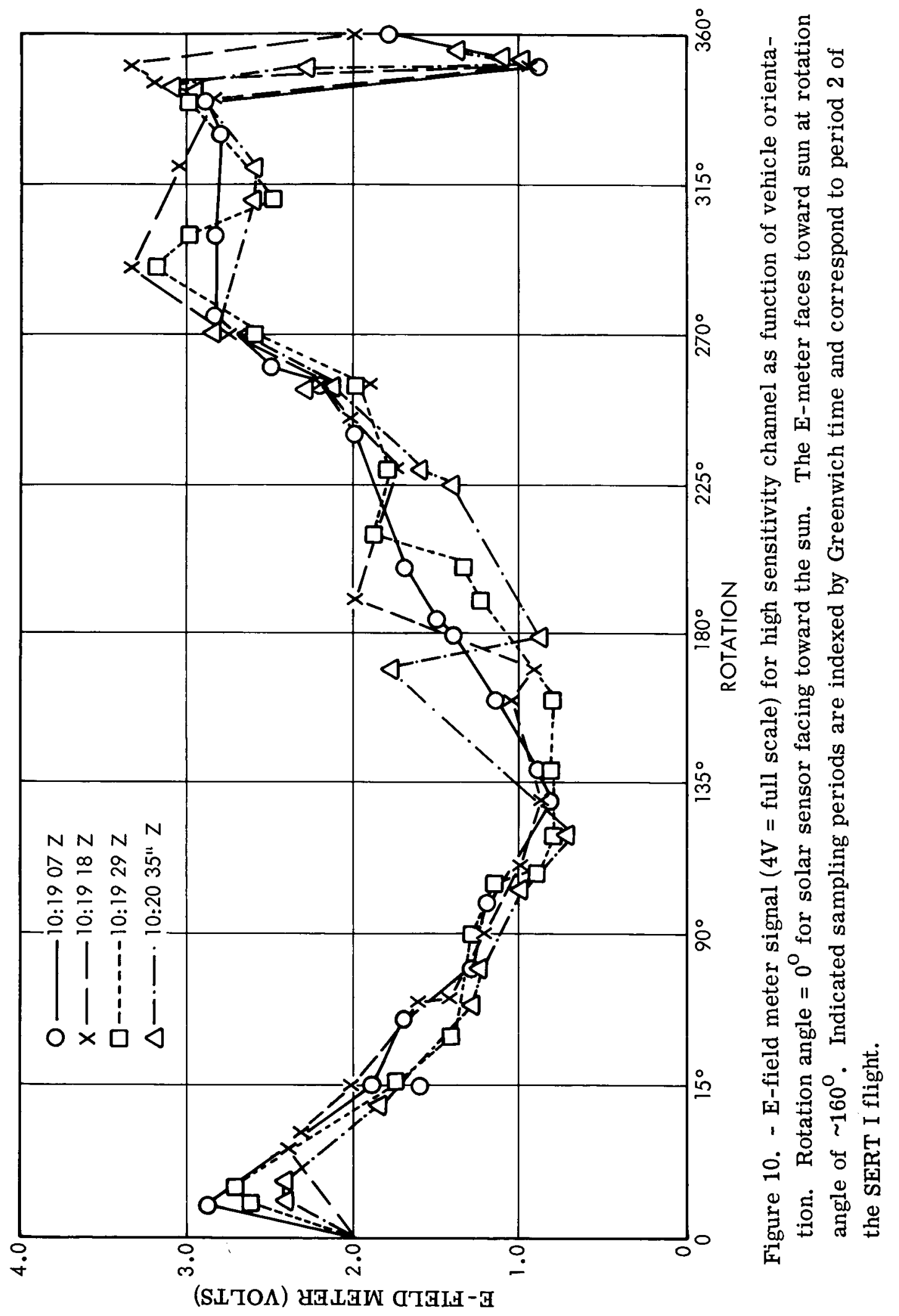




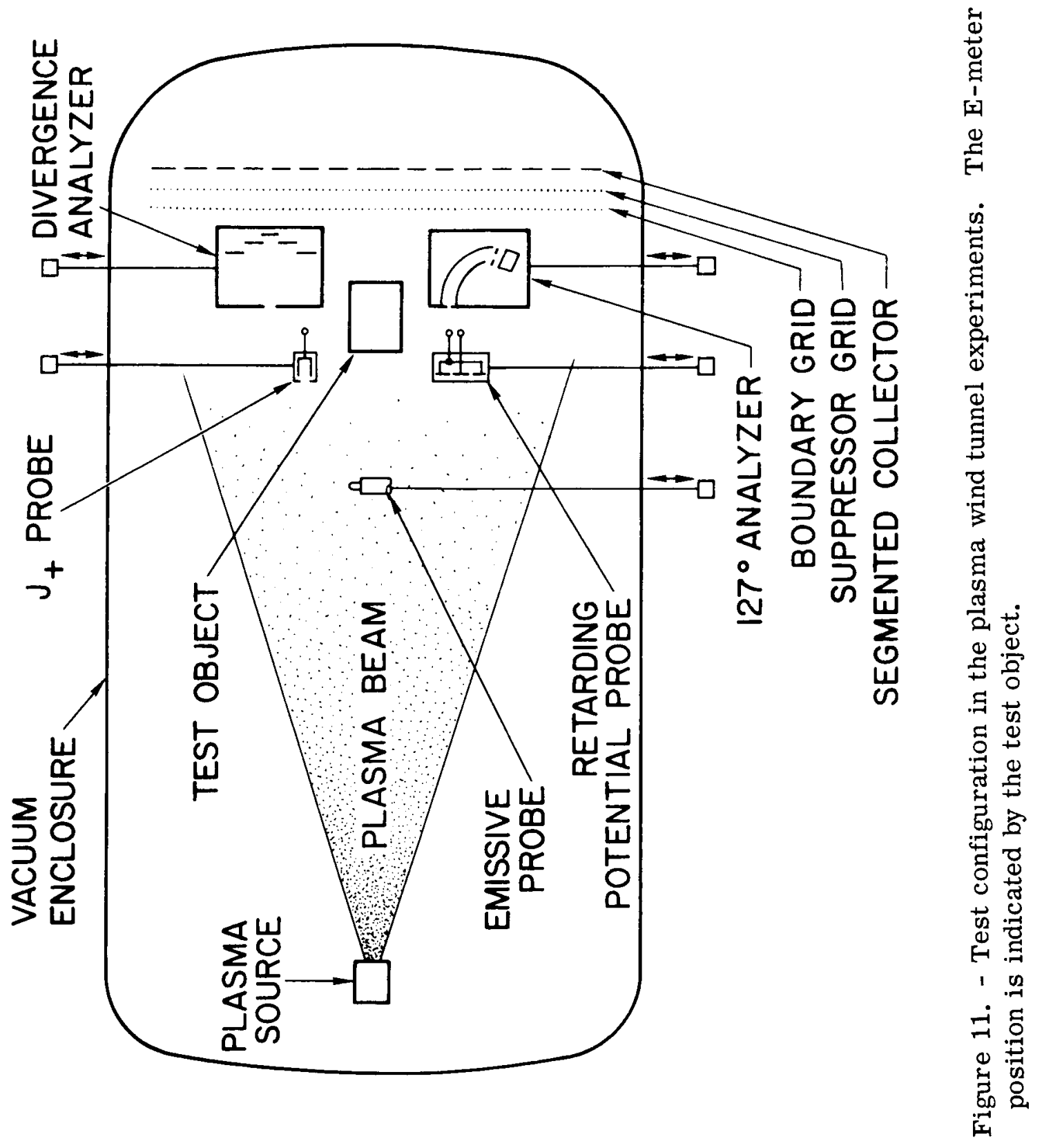



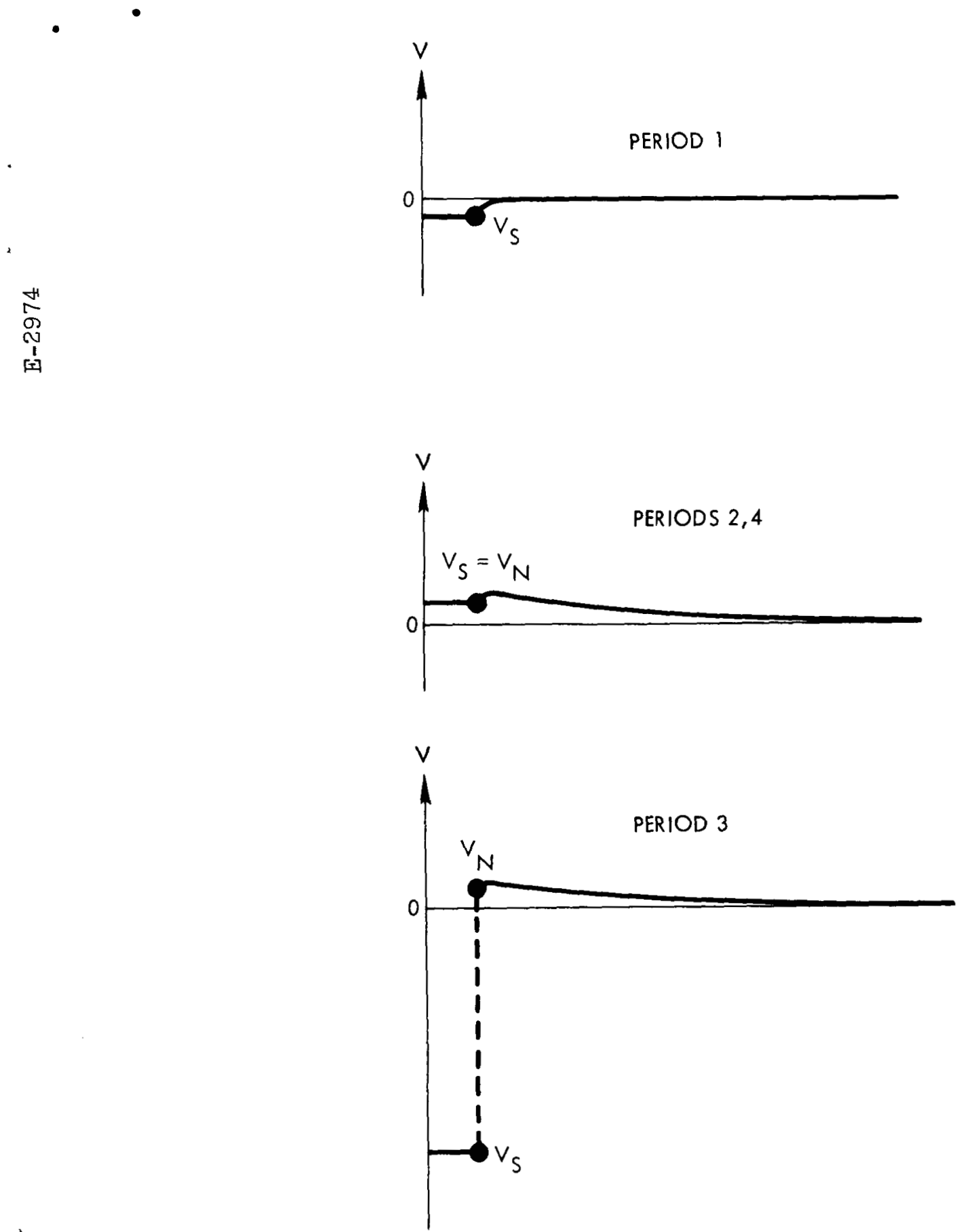

Figure 12. - Model of potential configuration during flight test. Plasma thrust beam is indicated by the curve from the dot outward to the potential of the ambient plasma $(\mathrm{V}=0)$. Vehicle potential $\mathrm{V}_{\mathrm{S}}$ is indicated by the short horizontal bar. 